\title{
Contrast-enhanced magnetic resonance angiography of the great arteries in patients with congenital heart disease: an accurate tool for planning catheter-guided interventions
}

\author{
Valsangiacomo Büchel, Emanuela R ; Di Bernardo, Stefano ; Bauersfeld, Urs ; Berger, Felix
}

\begin{abstract}
Background: Catheter-guided interventions are increasingly used for relief of lesions in patients with congenital heart disease. Exact anatomical imaging with measurement of the vascular structures is crucial in the planning of such interventions. This can be provided non-invasively and without radiation by contrast-enhanced MR angiography (CE-MRA). Aim: To evaluate the accuracy of the measurements of the vessels obtained by CE-MRA in comparison to those obtained by conventional X-ray angiography (XRA). Methods: Measurements of the diameters of aorta and pulmonary arteries were performed retrospectively and blinded on the CE-MRA and XRA images, in comparable locations. The limits of agreement between the two methods were calculated. Results: Twenty-one CE-MRA and XRA were performed in 20 children with congenital heart disease, median age 4years (1day-13years), weight $18 \mathrm{~kg}$ $(3.2-74 \mathrm{~kg})$. The time interval between CE-MRA and XRA was $2.6 \pm 2.3$ months. A total of 98 measurements, 38 of the aorta and 60 of the pulmonary arteries were performed on the images obtained by each technique. The correlation between CE-MRA and XRA measurements was excellent, $\mathrm{r}=0.97, \mathrm{p}<$ 0.0001 . The mean difference between the two techniques was $0.018 \pm 1.1 \mathrm{~mm}$; the limits of agreement -2.14 and $+2.18 \mathrm{~mm}$. Similar agreement was found for measures of the aorta $(\mathrm{r}=0.97$, mean difference $0.20 \pm 1.08 \mathrm{~mm})$ and of the pulmonary arteries $(\mathrm{r}=0.97$, mean difference $0.048 \pm 0.89 \mathrm{~mm})$. Conclusions: CE-MRA provides accurate quantitative anatomical information, which highly agrees with XRA data, and can therefore be used for planning catheter-guided procedures
\end{abstract}

DOI: https://doi.org/10.1007/s10554-004-4017-y

Posted at the Zurich Open Repository and Archive, University of Zurich

ZORA URL: https://doi.org/10.5167/uzh-156344

Journal Article

Published Version

Originally published at:

Valsangiacomo Büchel, Emanuela R; Di Bernardo, Stefano; Bauersfeld, Urs; Berger, Felix (2005). Contrastenhanced magnetic resonance angiography of the great arteries in patients with congenital heart disease: an accurate tool for planning catheter-guided interventions. International Journal of Cardiovascular Imaging, 21(2-3):313-322.

DOI: https://doi.org/10.1007/s10554-004-4017-y 


\title{
Contrast-enhanced magnetic resonance angiography of the great arteries in patients with congenital heart disease: an accurate tool for planning catheter-guided interventions
}

\author{
Emanuela R. Valsangiacomo Büchel, Stefano DiBernardo, Urs Bauersfeld \& Felix Berger \\ University Children's Hospital, Zurich Division of Paediatric Cardiology, Switzerland
}

Received 19 May 2004; accepted in revised form 27 September 2004

Key words: angiography, catheter-guided interventions, congenital heart disease, magnetic resonance imaging

\begin{abstract}
Background: Catheter-guided interventions are increasingly used for relief of lesions in patients with congenital heart disease. Exact anatomical imaging with measurement of the vascular structures is crucial in the planning of such interventions. This can be provided non-invasively and without radiation by contrastenhanced MR angiography (CE-MRA). Aim: To evaluate the accuracy of the measurements of the vessels obtained by CE-MRA in comparison to those obtained by conventional X-ray angiography (XRA). Methods: Measurements of the diameters of aorta and pulmonary arteries were performed retrospectively and blinded on the CE-MRA and XRA images, in comparable locations. The limits of agreement between the two methods were calculated. Results: Twenty-one CE-MRA and XRA were performed in 20 children with congenital heart disease, median age 4 years (1 day-13 years), weight $18 \mathrm{~kg}(3.2-74 \mathrm{~kg})$. The time interval between CE-MRA and XRA was $2.6 \pm 2.3$ months. A total of 98 measurements, 38 of the aorta and 60 of the pulmonary arteries were performed on the images obtained by each technique. The correlation between CE-MRA and XRA measurements was excellent, $\mathrm{r}=0.97, p<0.0001$. The mean difference between the two techniques was $0.018 \pm 1.1 \mathrm{~mm}$; the limits of agreement -2.14 and $+2.18 \mathrm{~mm}$. Similar agreement was found for measures of the aorta $(\mathrm{r}=0.97$, mean difference $0.20 \pm 1.08 \mathrm{~mm})$ and of the pulmonary arteries $(r=0.97$, mean difference $0.048 \pm 0.89 \mathrm{~mm})$. Conclusions: CE-MRA provides accurate quantitative anatomical information, which highly agrees with XRA data, and can therefore be used for planning catheter-guided procedures.
\end{abstract}

\section{Introduction}

The anatomy of congenital heart defects (CHD), either pre or postoperative, can be very complex. Imaging strategies continue to evolve and at present increasingly more information is gleaned non-invasively [1]. In routine clinical practice cross-sectional echocardiography has replaced cardiac catheterization as the preoperative imaging modality of choice for several patients with CHD [2]. In case of a limited acoustic window the anatomy can be well demonstrated by magnetic resonance imaging (MRI) [3]. Contrast-enhanced magnetic resonance angiography (CE-MRA) is a newly developed technique increasing the diagnostic power of cardiac MRI studies and gaining more and more importance in non-invasive cardiac imaging $[4,5]$. CE-MRA has been proven to be an 
accurate diagnostic tool for pulmonary and systemic venous anomalies as well as for aortic arch anomalies [6-10].

The number of diagnostic catheterization is drastically declining [2]. In contrast catheter-guided interventions are increasingly used as alternative to surgical therapy [11]. Measuring exactly the dimensions of the vessels or of the valve annulus may be crucial in planning and proceeding for surgery or for a catheter-guided procedure [12]. As cardiac MRI, including CE-MRA, is being performed as diagnostic exam for clinical decisionmaking and for planning the necessary procedures in an increasing number of patients, the vascular structures of interest could be preliminary measured on the CE-MRA images.

Aim of this study was to evaluate the accuracy of the measurements of the vessels obtained by
CE-MRA in comparison with those obtained by conventional X-ray angiography (XRA).

\section{Materials and methods}

\section{Patients}

Twenty consecutive children with CHD underwent both cardiac MRI and catheterization. Patient's characteristics are shown in Table 1. The median age was 4 years, with a range from 1 day to 13 years; the median weight $18 \mathrm{~kg}$, with a range from 3.2 to $74 \mathrm{~kg}$. One patient underwent CEMRA and XRA twice, at 3 and 5 months of age, respectively (case 4 ).

Echocardiographic evidence of a residual lesion or of a complex anatomical situation (case 1, 9, 14, 16 and 20) that required clearer anatomical

Table 1. Patient's characteristics, anatomical findings and interventions performed.

\begin{tabular}{|c|c|c|c|c|c|}
\hline Case & Age & Diagnosis & Angiographic findings & Catheterization & Intervention \\
\hline 1 & 1 day & $\begin{array}{l}\text { Situs inversus, } \\
\text { AV-VA-Discordance, PA }\end{array}$ & Bilateral PDA & Interventional & PDA stent \\
\hline 2 & 7 years & dTGA, after switch & LPA stenosis & Interventional & LPA stent \\
\hline 3 & 12 years & $\mathrm{CoA}$ & $\mathrm{CoA}$ & Interventional & Ao stent \\
\hline \multirow[t]{2}{*}{4} & 3 months & $\mathrm{PA} / \mathrm{VSD}$ & LPA stenosis & Interventional & LPA angioplasty \\
\hline & 5 months & $\mathrm{PA} / \mathrm{VSD}$ & LPA stenosis & Interventional & LPA stent \\
\hline 5 & 10 months & TA, TGA, after BCPC & Residual CoA & Interventional & Ao angioplasty \\
\hline 6 & 8 years & TOF after repair & Multiple PPS & Diagnostic & None \\
\hline 7 & 13 years & CoA, Ao graft & Aograft stenosis & Diagnostic & None \\
\hline 8 & 9 years & dTGA, after switch & RPA stenosis & Interventional & RPA stent \\
\hline 9 & 1 year & DORV, single RV, after BCPC & BCPC open & Diagnostic & None \\
\hline 10 & 9 months & $\mathrm{PA} / \mathrm{VSD}$ & LPA stenosis & Interventional & LPA Angioplasty \\
\hline 11 & 3 weeks & PA/VSD & $\begin{array}{l}\text { CE-MRA and CXA } \\
\text { not concordant }\end{array}$ & Diagnostic & None \\
\hline 12 & 7 months & IAA after repair & LPA stenosis & Interventional & LPA angioplasty \\
\hline 13 & 9 years & $\mathrm{CoA}$ after repair & Residual CoA & Interventional & Ao angioplasty \\
\hline 14 & 4 years & DORV, TGA, PS, MV straddling & Known anatomy & Interventional & Atrial septostomy \\
\hline 15 & 12 years & dTGA, after switch & LPA stenosis & Interventional & LPA stent \\
\hline 16 & 4 years & Ebstein, after BCPC & BCPC open & Diagnostic & None \\
\hline 17 & 9 years & CoA after repair & Residual CoA & Interventional & Ao stent \\
\hline 18 & 11 years & dTGA, after switch & LPA stenosis & Interventional & LPA stent \\
\hline 19 & 14 years & $\mathrm{PA} / \mathrm{VSD}$ & Multiple PPS & Interventional & Angioplasty, stent \\
\hline \multirow[t]{2}{*}{20} & 5 months & LAI, AVSD, singleRV, & Known anatomy & Diagnostic & none \\
\hline & & PS, SV anomalies & RPA stenosis & & \\
\hline
\end{tabular}

AVSD, atrioventricular septal defect; Ao, aorta; AV, atrio-ventricular; $\mathrm{BCPC}$, bidirectional cavopulmonary connection; CoA, aortic coarctation; dTGA, d- transposition of the great arteries; DORV, double outlet right ventricle; Ebstein, Ebstein malformation; IAA, interrupted aortic arch; LAI, left atrial isomerism; LPA, left pulmonary artery; MV, mitral valve; PA/VSD, pulmonary atresia with ventricular septal defect; PDA, patent ductus arteriosus; PS, pulmonary stenosis; PPS, peripheral pulmonary stenosis; RPA, right pulmonary artery; RV, right ventricle; SV, systemic veins; TA, tricuspid atresia; TOF, tetralogy of Fallot; VA, ventriculo-arterial. 
delineation was the indication for performing CEMRA. In 15 patients an interventional procedure was planned based on the CE-MRA findings. In 6 cases a diagnostic catheterization was additionally performed, for measurement of the pulmonary vascular resistance in 4 (case 6, 9, 16 and 20), for anatomical imaging before surgery in one (case 7) and to rule out a residual lesion needing reintervention in another one (case 11).

\section{Technique}

\section{$C E-M R A$}

The contrast-enhanced angiographies were all performed with a 1.5T GE Signa MRI/I Echospeed scanner (GE Medical systems, Milwaukee, Wisconsin, USA) by using a phased-array cardiac coil. CE-MRA was performed using a 3D-fast spoiled gradient refocused echo sequence in the coronal plane with the following parameters: TE minimum, flip angle $30^{\circ}$, matrix $156 \times 160$, bandwidth $62.5 \mathrm{kHz}$, number of excitations 1 , field of view $26-42 \mathrm{~cm}$ depending on body size, slice thickness $3.2-4 \mathrm{~mm}$ and 20-32 partitions as appropriate for covering the region of interest in an adequate time, linear $\mathrm{k}$-space filling. Scanning time for each sequence ranged from 10 to $18 \mathrm{~s}$. Whenever possible, depending on the cooperation of the patient images were acquired during breath hold. Three consecutive sets of images were acquired to visualize all the arteries and the veins of both the systemic and the pulmonary circulation. The timing of image acquisition, depending on the vessel of interest, was determined by using the testbolus technique; the arrival of the contrast medium in the vessel of interest and its maximal contrast effect were visualized on real time images [13]. This was performed by visual inspection (qualitatively) after injection of $2 \mathrm{ml}$ Gadodiamide (Omniscan ${ }^{\circledR}$, Nycomed AG, Wädenswil, Switzerland). Contrast medium was injected as a bolus via peripheral intravenous access with a dose of $0.3 \mathrm{mmol} / \mathrm{kg}$ body weight by using an automated contrast injector at injection rate of $2 \mathrm{ml} / \mathrm{s}$ followed by a saline flush of $20 \mathrm{ml}$.

CE-MRA source data were reconstructed on a commercially available workstation (Advantage Window 4.0; GE Medical systems, Milwaukee,
Wisconsin, USA) with a maximum intensity projection algorithm. The course of the vessels was reconstructed in every desired plane by using multiplanar reformation and $3 \mathrm{D}$ volume rendering techniques. Measurements of the vessels were performed on the images obtained by multiplanar reformation. Volume rendering images were used only for advanced understanding of the topographic vascular anatomy.

\section{$X R A$}

Conventional angiographies were performed during cardiac catheterization with a biplane Integris BH 5000 cardiovascular system (Philips Medical System, Best, The Netherlands). Hemodynamic measurements were obtained in appropriate locations depending on the cardiac malformation, such as the cardiac chambers and the main venous and arterial vessels. Specific angiographies were acquired for anatomic delineation before catheterguided intervention in 15 and for diagnostic purposes in 6 patients. Angiographies were obtained by injecting a ionized contrast medium (Iopamiro ${ }^{\circledR}$, Sintetica SA, Mendrisio, Switzerland) in a dose of $1-1.5 \mathrm{ml} / \mathrm{kg}$ and with the maximal flow velocity allowed by the size of the catheter, i.e. 1$2 \mathrm{ml} / \mathrm{s}$, by using a power injector. The images were acquired with a frame rate of $25 / \mathrm{s}$. The imaging plane was chosen depending on the vascular structures needing to be visualized, in the standard projections that have been previously described [12]. If an intervention was planned, measurements of the vessels were performed directly on the screen, by using catheter calibration. The size of the catheters used ranged from 4 to 6 French.

\section{Data analysis}

Two experienced investigators (ERV and SDB), blinded to each other, retrospectively measured the diameter of the vessels in several comparable locations (Table 2) on the images obtained by CEMRA (ERV) and XRA (SDB), respectively. The underlying cardiovascular lesion determined which vessels could be measured. Thus in patients who were evaluated for stenosis of the pulmonary arteries, the diameter of the pulmonary arteries was measured at the narrowest point of stenosis, 
Table 2. Locations of the measurements performed on the CE-MRA and XRA images.

\begin{tabular}{ll}
\hline Location & $n$ \\
\hline LPA & \\
Origin & 16 \\
Before branching & 15 \\
RPA & \\
Origin & 13 \\
Before branching & 12 \\
RVOT & 2 \\
Subvalvar & 3 \\
At valve annulus & 1 \\
Supravalvar & 1 \\
Aortic root & 7 \\
Ascending aorta & \\
Aortic arch & 8 \\
Transverse & 5 \\
Distal & 8 \\
Isthmus & 4 \\
Descending aorta & 3 \\
Aortic conduit & \\
\hline
\end{tabular}

LPA, left pulmonary artery; RPA, right pulmonary artery; RVOT, right ventricular outflow tract.

corresponding usually to the origin of the vessel, and further peripherally where the dimension was wider again. In patients with lesions of the aortic arch, the aortic arch was measured in standard locations, including the ascending aorta, the transverse aortic arch, between brachiocephalic trunk und left carotid artery, the distal aortic arch, between right carotid artery and right subclavian artery, the isthmus and the thoracic aorta at the level of the diaphragm.

Information was shared about the vessel and the location where the measurements should be performed, but not about the results of such measurements. The CE-MRA images were reconstructed as described above in order to obtain similar projections as the XRA images; the diameters of the vessels were then measured directly by using the intrinsic electronic calibration of the system. XRA were retrospectively reviewed by using Inturis Cardio View software, Release 1.2 (Philips Medical Systems, Best, The Netherlands). Before measuring the diameter of the vessels, calibration was obtained by comparing the diameter of the catheter shown on the angiographic images with the catheter diameter in French supplied by the manufacturer. In order to increase the accuracy of the measurements the external diameter of the catheter was measured when empty of contrast medium.

\section{Statistics}

Numerical data are reported as mean values and standard deviations or as median values and ranges where appropriate. The measures obtained were compared to each other by Pearson's correlation and by calculating the Bland Altman limits of agreement [14]. A $p$ value $<0.05$ was considered as statistically significant. The study was approved by the Research Ethics Board of our institution.

\section{Results}

Twenty-one CE-MRA and XRA were performed in 20 patients. The mean time interval between CE-MRA and XRA was $2.6 \pm 2.3$ months. CEMRA was performed in 10 conscious children, in general anesthesia in 9 and in sedation in 2. All XRA were performed during cardiac catheterization and in general anesthesia. No procedural complications were observed.

\section{Anatomy}

CE-MRA and XRA showed similar morphologic findings in 19 patients (Figure 1). In a newborn girl with situs inversus, atrio-ventricular and ventriculo-arterial discordance and pulmonary atresia with non-confluent pulmonary arteries that were supplied by a bilateral ductus arteriosus (case 1), the correct diagnose was first depicted at CEMRA and successively confirmed at XRA. In this case an adequate management consisting in stenting both ductus arteriosus could be planned and performed on the basis of the anatomical information obtained by CE-MRA. In one patient (case 11) CE-MRA and XRA did not show the same anatomical findings (Figure 2). In this child with pulmonary atresia with ventricular septal defect, Blalock-Taussig shunt to the pulmonary arteries confluence and atypical patent ductus arteriosus to the left pulmonary artery, a severe 

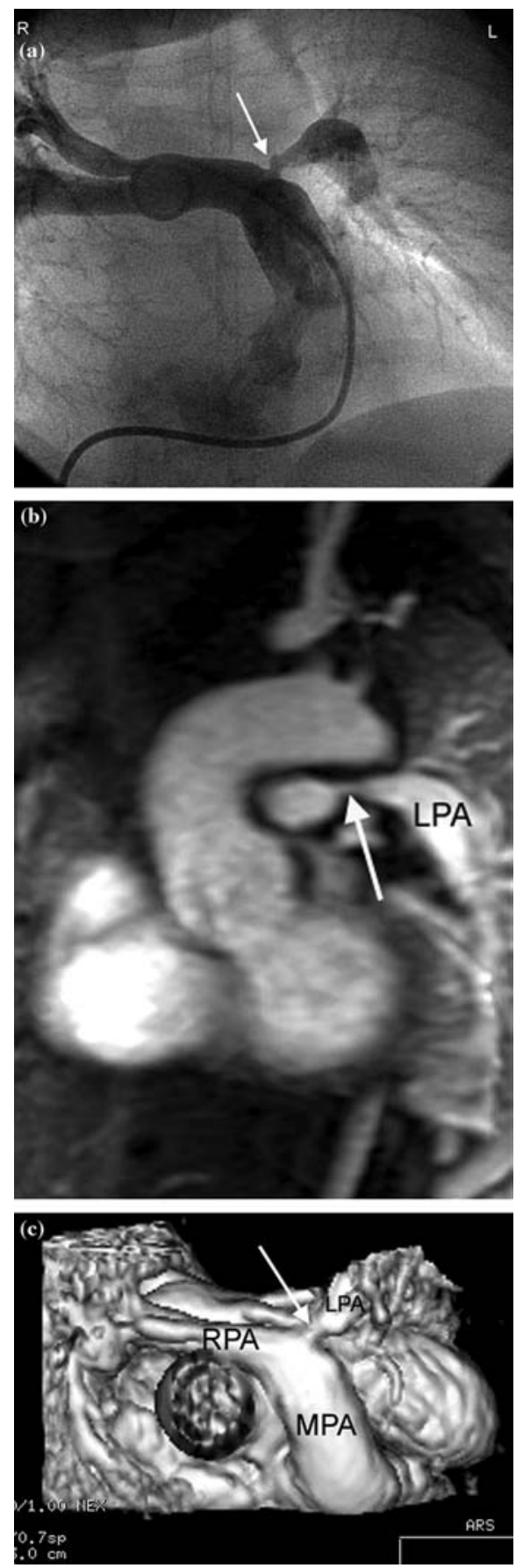

Figure 1. Severe Stenosis of the left pulmonary artery as demonstrated by XRA (a) and by CE-MRA with the multi intensity projection reconstruction (b) and in the $3 \mathrm{D}$ reconstruction (c). The diameter of the narrowest segment was $2.7 \mathrm{~mm}$ on the CXA images and $2.5 \mathrm{~mm}$ on the CE-MRA images. stenosis of the left pulmonary artery was diagnosed at CE-MRA, but not confirmed at XRA. In two patients (case 6 and 18) who previously underwent angioplasty of the left pulmonary artery, XRA depicted a small aneurysm at the origin of the vessel, which had not been described by CEMRA.

\section{Quantitative measurements}

Ninety-eight single measurements were performed on the CE-MRA and XRA images, respectively. Fifty-four measures were performed on the pulmonary arteries side branches, 35 on the aorta (Figure 2), 6 on the right ventricular outflow tract and 3 on an aortic conduit. Table 2 summarized the exact anatomical location of the measurements. For statistical analysis measures of the right ventricular outflow tract were included in the group of the pulmonary arteries and measures of the aortic conduit in the group of the aorta. The mean diameters of the pulmonary arteries measured $7.92 \pm 3.87 \mathrm{~mm}$ on the CE-MRA images and $7.87 \pm 3.56 \mathrm{~mm}$ on the XRA images. The mean diameter of the aorta was $10.65 \pm 4.27 \mathrm{~mm}$ on the CE-MRA images and $10.76 \pm 4.35 \mathrm{~mm}$ on the XRA images. There was an excellent correlation between the measurements performed on the CEMRA and those performed on the XRA images (Figure 3). The Bland Altman limits of agreements between both methods are described in Table 3 and illustrated in Figure 4. Similar agreement was found for measures of the aorta $(r=0.97$, mean difference $0.20 \pm 1.08 \mathrm{~mm}$ ) and of the pulmonary arteries $(r=0.97$, mean difference $0.048 \pm 0.89 \mathrm{~mm})$.

\section{Discussion}

The recent evolution in diagnosing CHD indicates a clear trend toward the non-invasive approach. Cross-sectional echocardiography and MRI are at present the leading techniques for advanced anatomical and functional imaging. Diagnostic catheterization with conventional angiography is limited to selected cases, in whom pulmonary vascular resistance need to be determined or specific anatomical conditions have to be addi- 

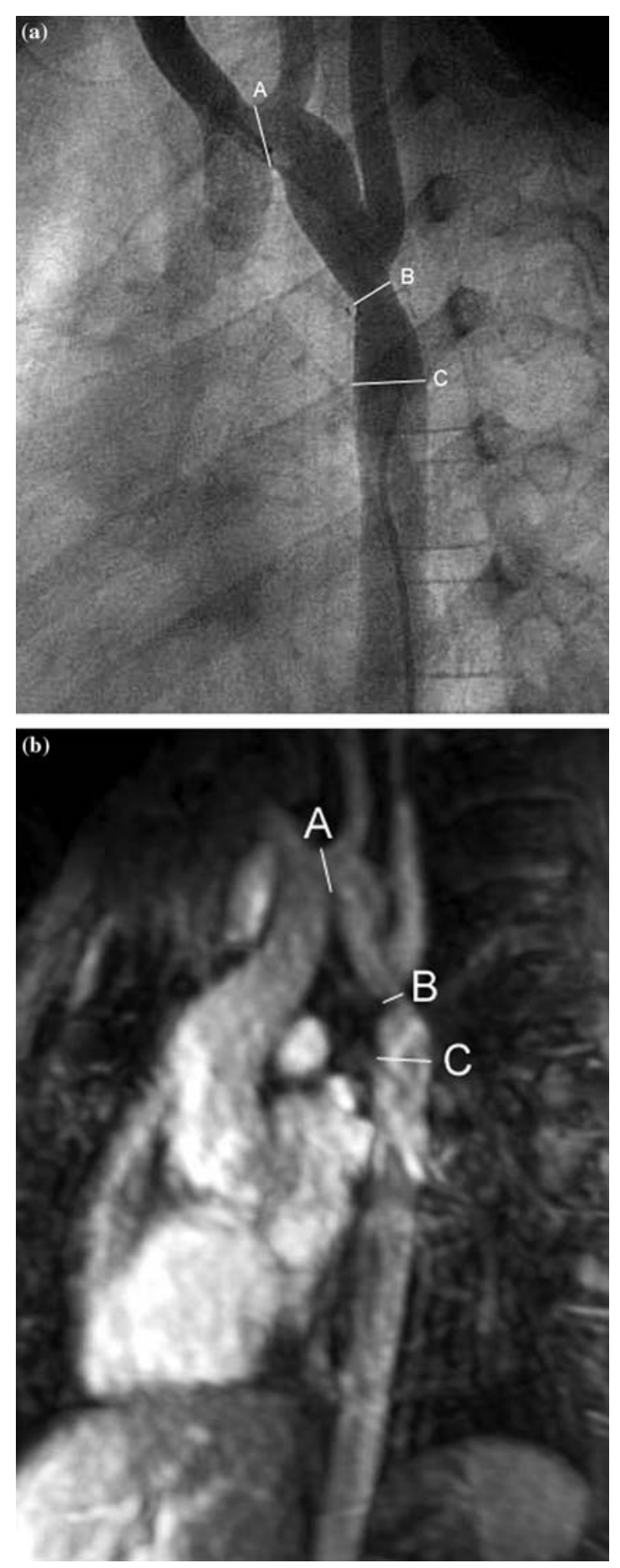

Figure 2. Comparative measurements of the aortic arch in a patients with a residual aortic coarctation on the XRA images (a) and on the CE-MRA images (b).

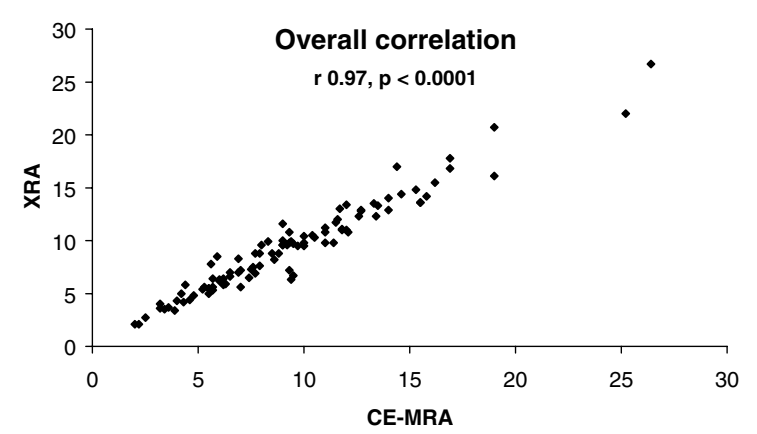

Figure 3. Pearson's correlation between the XRA and the CE-MRA measurements.

Table 3. Bland Altman limits of agreement between CEMRA and CXA measurements.

\begin{tabular}{lllll}
\hline & $n$ & $\begin{array}{l}\text { Mean } \\
\text { difference }\end{array}$ & $\begin{array}{l}\text { SD } \\
\text { difference }\end{array}$ & $\begin{array}{l}\text { Limits of } \\
\text { agreement }\end{array}$ \\
\hline All & 98 & 0.01837 & 1.104 & $-2.145 /+2.182$ \\
$\quad$ measurements & & & \\
Aorta & 38 & 0.2091 & 1.086 & $-2.338 /+1.92$ \\
$\begin{array}{l}\text { Pulmonary } \\
\quad \text { arteries }\end{array}$ & 60 & 0.04815 & 0.8952 & $-1.706 /+1.803$ \\
\hline
\end{tabular}

tionally clarified [2]. In contrast catheter-guided interventions are increasingly performed as alternative to surgery. Measuring exactly the dimensions of vascular structures that need to be treated is an important feature in diagnostic studies and interventional procedures [12].

This study assessed the accuracy of measurements of the diameter of the vessels performed on images obtained by CE-MRA compared with those obtained by XRA, considered as the gold standard, and demonstrated the accuracy of such measurements. We found an excellent correlation between the CE-MRA and the XRA dimensions. Our results validate the technique not only for larger patients, but also for very young children, being 8 of the 20 patients studied infants younger than 1 year. This proves that this technique is robust independently of the age of the patients and of the size of the vessels. Infants represent the patient group that mostly may take advantage from an accurate non-invasive diagnostic assessment. In fact children who underwent neonatal repair for complex CHD may present a few 

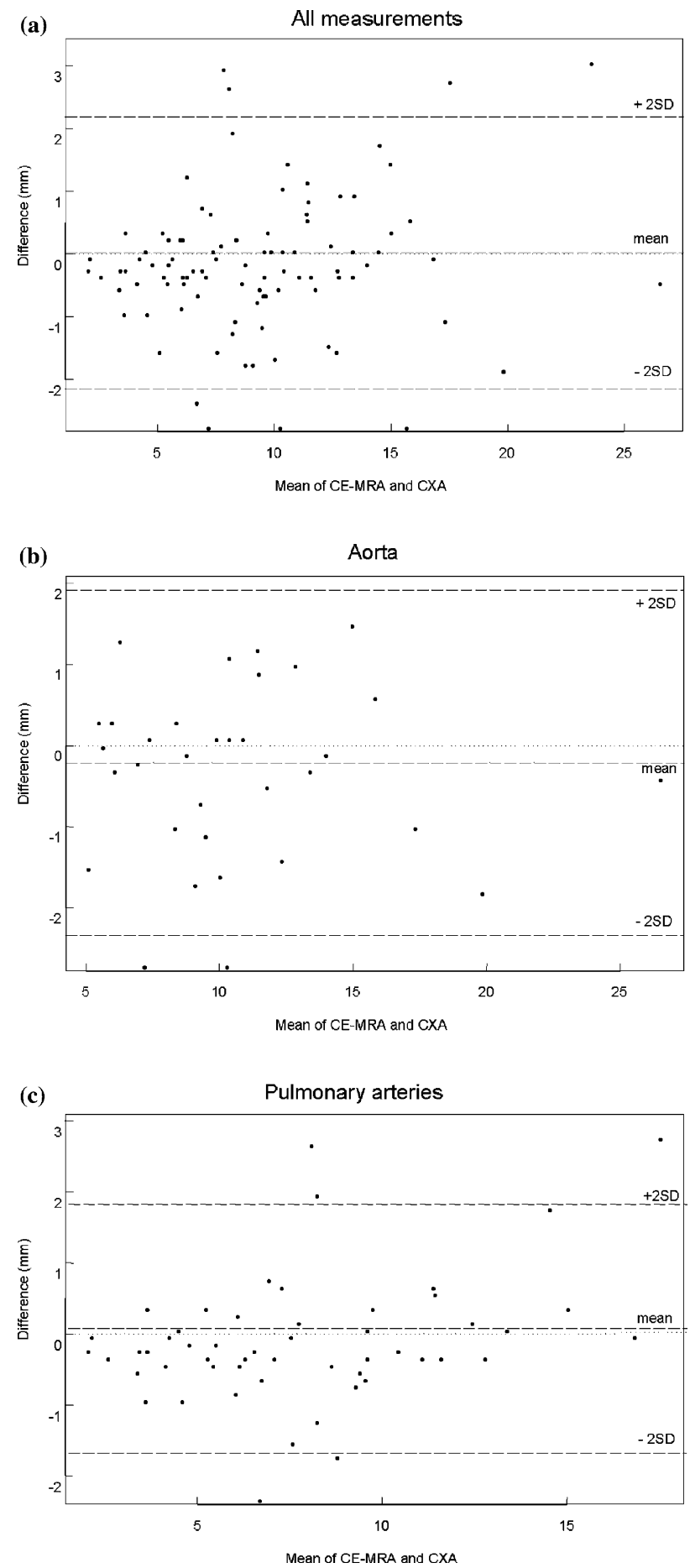

Figure 4. Bland Altman limits of agreement for all measurements performed (a) and for the measurements of the aorta (b) and of the pulmonary arteries (c). 
months later with significant residual findings, necessitating relief by catheter-guided interventions.

The clinical relevance of our data consists in the potential of avoiding performing a diagnostic catheterization or additional XRA before an intervention. As cardiac catheterization is associated with well-known risks, several advantages of CE-MRA in comparison with XRA are evident [15]. CE-MRA is non-invasive, sedation time is shorter, no particular additional expertise is required for the examination of small children and no arterial puncture is needed, what preserves central vascular access. The lack of ionising radiation may be one of the most striking advantages of this technique. Long-term health effects, such as fatal malignancies and teratogenic effects have been described in patients irradiated during diagnostic or interventional catheterization [16]. Since children are at higher risk than adults for developing a solid tumour and a substantial number of patients with CHD may undergo repeated cardiac catheterization, leading to a higher cumulative Xray dose, to reduce radiation is crucial in these patients [17, 18]. A fast and efficient catheter-guided intervention can be achieved by accurately planning such intervention on the basis of the images and measurements obtained by CE-MRA. Moreover, the renal function of critically ill patients may be preserved by reducing the amount of iodinated contrast-medium injected, as the contrast medium used for CE-MRA is not nephrotoxic $[19,20]$.

For ethical reasons we could not measure systematically every vessel in each patient, as XRA was performed selectively only in the vessels of clinical interest in order to reduce radiation to the patient. Thus our results can be divided for two groups of patients: children with lesions of the pulmonary arteries and children with anomalies of the aortic arch. Even if statistically not significant the differences observed were slightly larger for measurements of the aorta than for the pulmonary arteries. Vonder Muhll et al. [21] recently reported a similar slightly lower correlation for measurements of the aorta. The different degree of pulsation in the high-pressure (aorta) and in the lowpressure vessels (pulmonary arteries) may be the reason for this observation. In fact on XRA images the dimensions are usually measured during the end-systolic phase, when the vessel is maximally distended. Images obtained by CE-MRA are a summation of data acquired during all cardiac phases and therefore the dimensions represent an average diameter of the vessel, smaller than in the end-systolic phase. The higher frame rate of image acquisition and therefore higher temporal resolution of XRA may enhance this observation. In spite of this technical difference in image acquisition, our results demonstrate the accuracy of this promising technique.

In one single case the anatomical information obtained by CE-MRA did not correspond to the findings shown by XRA. In presence of complex vascular anatomy with abnormal blood flow distribution, a situation mostly occurring in the pulmonary arteries, when surgical shunts, ductus arteriosus and aortopulmonary collaterals contribute to blood flow, particular streaming effects may represent a diagnostic pitfall in the interpretation of CE-MRA images. These different sources of blood flow can compete to each other, causing loss of contrast in the vessel on the CE-MRA images, which may mimicry a stenosis of the vessel. If such situation is recognized the acquisition of more temporal phases during CE-MRA and the careful examination of all images in every different phase, when the contrast medium may be steadily distributed in all vessels, is recommended.

The recent advances in MRI technology with implementation of high performance gradient systems and parallel imaging has improved both spatial and temporal resolution of CE-MRA by maintaining a good image quality [22-24]. Further technological improvements are allowing to start performing MRI-guided interventional procedures based on real time MR-images [25]. In this context our results may also indicate that MRI-guided interventions can be accurately and safely planned on images obtained by CE-MRA, without need for a previous XRA.

This study shows the limitations of being retrospective. The calibration method for measurements on the XRA images for correction of magnification, by using the injection catheter as correction factor, may represent a well-known 
source of error, but it is still used as sufficient calibration tool during clinical practice. More accurate would be calibration using a sphere of known size and volume [12]. The time interval between both examinations, CE-MRA and XRA, may be another potential source of difference between the measurements.

\section{Conclusion}

Measurements of the vascular dimensions performed on CE-MRA images are very accurate when compared to XRA measurements and can therefore be utilized for planning catheter-guided interventions. Performing previous diagnostic evaluation by CE-MRA allows reducing the amount of ionising radiation and of iodinated contrast medium used during interventional catheterization. Particularly in small children with complex CHD anatomical evaluation by CEMRA should be considered before performing any interventional procedure on the great arteries.

\section{Acknowledgement}

We thank Dr.L.Molinari for the statistical support he kindly provided.

\section{References}

1. Pfammatter JP, Berdat P, Haemmerli M, Carrel T. Pediatriccardiac surgery after exclusively echocardiographicbased diagnostic work-up. Int J Cardiol 2000; 74: 185190.

2. Russell J, Justino H, Dipchand A, Yoo SJ, Kim YM, Freedom RM. Noninvasive imaging in congenital heart disease. Curr Opin Cardiol 2000; 15: 224-237.

3. Holmqvist C, Larsson EM, Stahlberg F, Laurin S. Contrast-enhanced thoracic 3D-MR angiography in infants and children. Acta Radiol. 2001; 42: 50-58.

4. Rebergen SA, de Roos A. Congenital heart disease. Evaluation of anatomy and function by MRI. Herz 2000; 4: 365383.

5. Greil GF, Powell AJ, Gildein HP, Geva T. Gadoliniumenhanced three-dimensional magnetic resonance angiography of pulmonary and systemic venous anomalies. J Am Coll Cardiol 2002; 39: 335-341.
6. Masui T, Katayama M, Kobayashi S, et al. Gadoliniumenhanced MR angiography in the evaluation of congenital cardiovascular disease per-and postoperative states in infants and children. J Magn Reson Imaging 2000; 12: 1034-1042.

7. Valsangiacomo ER, Levasseur S, McCrindle BW, MacDonald C, Smallhorn JF, Yoo SJ. Contrast-enhanced MR angiography of pulmonary venous abnormalities in children. Pediatr Radiol 2003; 33: 92-98.

8. Prasad SK, Soukias N, Hornung T, et al. Role of magnetic resonance angiography in the diagnosis of major aortopulmonary collaterals and partial anomalous pulmonary venous drainage. Circulation 2004; 109: 207-214.

9. Kondo C, Takada K, Yokoyama U, Nakajima Y, Momma K, Sakai F. Comparison of three-dimensional contrast-enhanced magnetic resonance angiography and axial radiographic angiography for diagnosing congenital stenoses in small pulmonary arteries. Am J Cardiol 2001; 87: 420-424.

10. Weinberg PM, Hubbard AM, Fogel MA.Aortic arch and pulmonary arteries anomalies in children. Semin Roentg 1998; 33: 262-280.

11. Rao PS. Interventional pediatric cardiology: state of the art and future directions. Pediatr Cardiol 1998; 19: $107-124$.

12. Culham JAG. Physical principles of image formation and projections in angiocardiography. In: Freedom RM, Mawson JB, Yoo SJ, Benson LN. Congenital Heart Disease. Textbook of Angiocardiography.Armonk NY: Futura, 1997: 39-93.

13. Neimatallah MA, Ho VB, Dong Q, Williams D, Patel S, Song JH et al. Gadolinium-enhanced 3D magnetic resonance angiography of the thoracic vessels. J Magn Reson Imaging 1999; 10: 758-770.

14. Bland JM, Altman DG. Statistical methods for assessing agreement between two methods of clinical measurement. Lancet 1986; 1(8476): 307-310.

15. Vitiello R, McCrindle BW, Nykanen D, Freedom RM, Benson LN. Complications associated with pediatric cardiac catheterization. J Am Coll Cardiol 1998; 32: 14331440.

16. Kovoor P, Ricciardello M, Collins L, Uther JB, Ross DL. Risk to patients from radiation associated with radiofrequency ablation for supraventricular tachycardia. Circulation 1998; 98: 1534-1540.

17. ICRP 60.1990 Recommendations of the International Commission on Radiological Protection. Ann ICRP 1990; 21: $1-3$.

18. Schueler BA, Julsrud PR, Gray JE, Stears JG, Wu KY. Radiation exposure and efficacy of exposure-reduction techniques during cardiac catheterization in children. AJR 1994; 162: 173-177.

19. Niendorf HP, Haustein J, Cornelius I, Alhassan A, Clauss W. Safety of gadolinium-DTPA: extended clinical experience. Magn Reson Med 1991; 22: 229-232.

20. Goyen M, Debatin JF. Gadopentetate Dimeglumine-enhanced three-dimensional MR-angiography: dosing, safety, and efficacy. J Magn Reson Imaging 2004; 19: 261-273. 
21. Vonder Muhll IF, Babu-Narayan S, Mullen M, Mohiaddin R. Cardiac magnetic resonance imaging accurately predicts findings at angiography in patients undergoing coarctation stenting. J Cardiovasc Magn Reson 2004; 6: 300.

22. Fink C, Eichhorn J, Kiessling F, Bock M, Delorne S. Timeresolved multiphasic 3D MR angiography in the diagnosis of the pulmonary vascular system in children. Fortschr Roentgenstr 2003; 175: 929-935.

23. Balci NC, Yalcin Y, Tunaci A, Balci Y. Assessment of the anomalous pulmonary circulation by dynamic contrast-enhanced MR angiography in under four seconds. J Magn Reson Imaging 2003; 21: 1-7.
24. Goyen M, Laub G, Ladd ME, et al. Dynamic 3D angiography of the pulmonary arteries in under four seconds. $\mathbf{J}$ Magn Reson Imaging 2001; 13: 372-377.

25. Razavi R, Hill DLG, Keevil SF, et al. Cardiac catheteterisation guided by MRI in children and adults with congenital heart disease. Lancet 2003; 362: 1877-1882.

Address for correspondence: Emanuela R. Valsangiacomo Büchel MD, Division of Paediatric Cardiology, University Children's Hospital Zurich, Steinwiesstr.75, 8032 Zurich - Switzerland. Tel.: + 41-1-266-7382; Fax.: +41-1-266-7981

E-mail: emanuela.valsangiacomo@kispi.unizh.ch 